\title{
Measure of Departure from Point Symmetry and Decomposition of Measure for Square Contingency Tables
}

\author{
Kiyotaka Iki $^{1 * *}$, Sadao Tomizawa ${ }^{2}$ \\ ${ }^{1}$ Faculty of Economics, Nihon University, Chiyoda-ku, Tokyo, 101-8360, Japan \\ ${ }^{2}$ Faculty of Science and Technology, Tokyo University of Science, Noda City, Chiba, 278-8510, Japan
}

\section{ARTICLE INFO}

Article History

Received 07 Jul 2020

Accepted 21 Dec 2020

Keywords

Conditional symmetry

Global symmetry

Kullback-Leibler information

Measure

Point symmetry

Square contingency table

\section{ABSTRACT}

For square contingency tables with ordered categories, Tomizawa, Biometrica J. 28 (1986), 387-393, considered the conditional point symmetry model. Kurakami et al., J. Stat. Adv. Theory Appl. 17 (2017), 33-42, considered the another point symmetry and the reverse global symmetry model. The present paper proposes Kullback-Leibler information type measures to represent the degree of departure from each of the models. Also this paper shows a theorem that the measure for the another point symmetry model is equal to the sum of the measures for the reverse global symmetry model and for the conditional point symmetry model.

(c) 2021 The Authors. Published by Atlantis Press B.V. This is an open access article distributed under the CC BY-NC 4.0 license (http://creativecommons.org/licenses/by-nc/4.0/).

\section{INTRODUCTION}

For an $r \times r$ contingency table with the same row and column ordinal classifications, let $X$ and $Y$ denote the row and column variables, respectively. Also let $\operatorname{Pr}(X=i, Y=j)=p_{i j}(1 \leq i, j \leq r)$. The symmetry (S) model (Bowker, [1]) is defined by

$$
p_{i j}=p_{j i} \quad(i<j)
$$

see also Bishop et al. ([2], p.282). The S model indicates a structure of symmetry of the probabilities $\left\{p_{i j}\right\}$ with respect to the main diagonal of the table. The global symmetry (GS) model (Read, [3]) is defined by

$$
\delta_{U}=\delta_{L}
$$

where

$$
\begin{aligned}
& \delta_{U}=\sum_{i<j} p_{i j} \quad(=\operatorname{Pr}(X<Y)), \\
& \delta_{L}=\sum \sum_{i>j} p_{i j} \quad(=\operatorname{Pr}(X>Y)) .
\end{aligned}
$$

The conditional symmetry (CS) model (Read, [3]; McCullagh, [4]) is defined by

$$
p_{i j}=\delta p_{j i} \quad(i<j)
$$

where $\delta$ is unknown parameter; see also Agresti ([5], p.361) and Tomizawa [6]. We note that the CS model is also expressed as

$$
\operatorname{Pr}(X=i, Y=j \mid X<Y)=\operatorname{Pr}(X=j, Y=i \mid X>Y) \quad(i \neq j) .
$$


So, the CS model indicates the CS. A special case of this model obtained by putting $\delta=1$ is the $S$ model. Read [3] gave the theorem that the $\mathrm{S}$ model holds if and only if both the GS and CS models hold.

Wall and Lienert [7] defined the point symmetry (PS) model defined by

$$
p_{i j}=p_{i^{*} j^{*}} \quad(1 \leq i, j \leq r)
$$

where $i^{*}=r+1-i$ and $j^{*}=r+1-j$. This model indicates a structure of PS of the probabilities $\left\{p_{i j}\right\}$ with respect to the center cell when $r$ is odd or the center point when $r$ is even in square tables. Kurakami et al. [8] considered the another point symmetry (APS) model defined by

$$
p_{i j}=p_{i^{*} j^{*}} \quad(1 \leq i, j \leq r ; i+j \neq r+1) .
$$

The APS model has less restrictions than the PS model by excluding the restrictions imposed on reverse diagonal probabilities. Kurakami et al. [8] considered reverse global symmetry (RGS) model defined by

$$
\Delta_{U}=\Delta_{L}
$$

where

$$
\begin{aligned}
& \Delta_{U}=\sum_{i+j<r+1} \sum_{i j} \quad(=\operatorname{Pr}(X+Y<r+1)), \\
& \Delta_{L}=\sum_{i+j>r+1} \sum_{i j} \quad(=\operatorname{Pr}(X+Y>r+1)) .
\end{aligned}
$$

Tomizawa [9] considered the conditional point symmetry (CPS) model defined by

$$
p_{i j}=\tau p_{i^{*} j^{*}} \quad(i+j<r+1),
$$

where $\tau$ is unknown parameter. The CPS model indicates that

$$
\operatorname{Pr}(X=i, Y=j \mid X+Y<r+1)=\operatorname{Pr}\left(X=i^{*}, Y=j^{*} \mid X+Y>r+1\right) .
$$

Kurakami et al. [8] gave the theorem that the APS model holds if and only if the RGS and CPS models hold. For more details on contingency tables analysis, see also Rao [10], Mosteller [11] and Wang [12].

By the way, when a model does not hold, we are interested in measuring the degree of departure from the model. Tomizawa [13], Tomizawa [14] and Tomizawa and Saitoh [15] considered the measures which indicate the degree of departure from S, GS and CS, respectively. Tomizawa and Saitoh [15] gave the theorem that the measure from $S$ is equal to the sum of the measure from GS and the measure from CS. Now, we are interested in proposing measures which indicate the degree of departure from APS, RGS and CPS, and showing the theorem that the measure from APS is equal to the sum of the measure from RGS and the measure from CPS.

Section 2 proposes the new measures which represent the degree of departure from APS, RGS and CPS (denoted by $\Phi_{A P S}$, $\Phi_{R G S}$ and $\Phi_{C P S}$ ), and show that the value of $\Phi_{A P S}$ is equal to the sum of the value of $\Phi_{R G S}$ and the value of $\Phi_{C P S}$. Section 3 gives an approximate standard error and large-sample confidence intervals for the proposed measures. Section 4 describes the relationship between the proposed measures and likelihood ratio statistic. Section 5 gives an example. Section 6 provides some concluding remarks.

\section{MEASURES FROM MODELS AND DECOMPOSITION OF MEASURE}

We assume that $p_{i j}+p_{i^{*} j^{*}}>0$, for $1 \leq i, j \leq r ; i+j \neq r+1$. Let $\Delta=\Delta_{U}+\Delta_{L}$ and

$$
p_{i j}^{c}=\frac{p_{i j}}{\Delta} \quad(1 \leq i, j \leq r ; i+j \neq r+1)
$$

We propose the measure for indicating how degree the departure from the APS model is as follows:

$$
\Phi_{A P S}=\frac{1}{\log 2} I_{A P S}
$$

where

$$
\begin{aligned}
I_{A P S} & =\sum_{i+j \neq r+1} \sum_{i j} p_{i j}^{c} \log \frac{p_{i j}^{c}}{p_{i j}^{A P S}}, \\
p_{i j}^{A P S} & =\frac{p_{i j}^{c}+p_{i^{*} j^{*}}^{c}}{2} .
\end{aligned}
$$


Note that $I_{A P S}$ is the Kullback-Leibler information between $\left\{p_{i j}^{c}\right\}$ and $\left\{p_{i j}^{A P S}\right\}$. The measure $\Phi_{A P S}$ has characteristics that, (i) $0 \leq \Phi_{A P S} \leq 1$, (ii) $\Phi_{A P S}=0$ if and only if $p_{i j}=p_{i^{*} j^{*}}$ for $i+j<r+1$, and (iii) $\Phi_{A P S}=1$ if and only if $p_{i j}=0\left(\right.$ then $\left.p_{i^{*} j^{*}}>0\right)$ or $p_{i^{*} j^{*}}=0\left(\right.$ then $\left.p_{i j}>0\right)$ for $i+j<r+1$.

Next, assume that $\Delta_{U}+\Delta_{L}>0$. Let

$$
\Delta_{U}^{c}=\frac{\Delta_{U}}{\Delta}, \Delta_{L}^{c}=\frac{\Delta_{L}}{\Delta}
$$

We propose the measure for indicating how degree the departure from the RGS model is as follows:

$$
\Phi_{R G S}=\frac{1}{\log 2} I_{R G S}
$$

where

$$
I_{R G S}=\Delta_{U}^{c} \log \frac{\Delta_{U}^{c}}{1 / 2}+\Delta_{L}^{c} \log \frac{\Delta_{L}^{c}}{1 / 2}
$$

Note that $I_{R G S}$ is the Kullback-Leibler information between $\left\{\Delta_{U}^{c}, \Delta_{L}^{c}\right\}$ and $\{1 / 2,1 / 2\}$. The measure $\Phi_{R G S}$ has characteristics that, (i) $0 \leq$ $\Phi_{R G S} \leq 1$, (ii) $\Phi_{R G S}=0$ if and only if $\Delta_{U}=\Delta_{L}$, and (iii) $\Phi_{R G S}=1$ if and only if $\Delta_{U}=0\left(\right.$ then $\left.\Delta_{L}>0\right)$ or $\Delta_{L}=0\left(\right.$ then $\left.\Delta_{U}>0\right)$.

Moreover, assuming that $\Delta_{U}>0, \Delta_{L}>0$, and $p_{i j}+p_{i^{*} j^{*}}>0$ for $1 \leq i, j \leq r ; i+j \neq r+1$, we propose the measure for indicating how degree the departure from the CPS model is as follows:

$$
\Phi_{C P S}=\frac{1}{\log 2} I_{C P S}
$$

where

$$
\begin{aligned}
I_{C P S} & =\sum_{i+j \neq r+1} \sum_{i j} p_{i j}^{c} \log \frac{p_{i j}^{c}}{p_{i j}^{C P S}}, \\
p_{i j}^{C P S} & = \begin{cases}\frac{\Delta_{U}}{\Delta}\left(p_{i j}^{c}+p_{i^{*} j^{*}}^{c}\right) & (i+j<r+1), \\
\frac{\Delta_{L}}{\Delta}\left(p_{i j}^{c}+p_{i^{*} j^{*}}^{c}\right) & (i+j>r+1) .\end{cases}
\end{aligned}
$$

Note that $I_{C P S}$ is the Kullback-Leibler information between $\left\{p_{i j}^{c}\right\}$ and $\left\{p_{i j}^{C P S}\right\}$.

We obtain the theorem as follows:

Theorem 1. The value of $\Phi_{A P S}$ is equal to the sum of the value of $\Phi_{R G S}$ and the value of $\Phi_{C P S}$.

Proof. We see that

$$
\begin{aligned}
& \Phi_{A P S}-\Phi_{C P S} \\
& =\frac{1}{\log 2}\left\{\sum_{i+j<r+1} \sum_{i j}^{c}\left(\log \frac{p_{i j}^{c}}{p_{i j}^{A P S}}-\log \frac{p_{i j}^{c}}{p_{i j}^{C P S}}\right)+\sum_{i+j>r+1} \sum_{i j}^{c}\left(\log \frac{p_{i j}^{c}}{p_{i j}^{A P S}}-\log \frac{p_{i j}^{c}}{p_{i j}^{C P S}}\right)\right\} .
\end{aligned}
$$

For $i+j<r+1$, we see

$$
\log \frac{p_{i j}^{c}}{p_{i j}^{A P S}}-\log \frac{p_{i j}^{c}}{p_{i j}^{C P S}}=\log \frac{\Delta_{U}^{c}}{1 / 2} .
$$

For $i+j>r+1$, we see

$$
\log \frac{p_{i j}^{c}}{p_{i j}^{A P S}}-\log \frac{p_{i j}^{c}}{p_{i j}^{C P S}}=\log \frac{\Delta_{L}^{c}}{1 / 2} .
$$


From equations (1) and (2), we see

$$
\begin{aligned}
& \Phi_{A P S}-\Phi_{C P S} \\
= & \frac{1}{\log 2}\left(\sum_{i+j<r+1} \sum_{i j} p^{c} \log \frac{\Delta_{U}^{c}}{1 / 2}+\sum_{i+j>r+1} p_{i j}^{c} \log \frac{\Delta_{L}^{c}}{1 / 2}\right) \\
= & \frac{1}{\log 2}\left(\Delta_{U}^{c} \log \frac{\Delta_{U}^{c}}{1 / 2}+\Delta_{L}^{c} \log \frac{\Delta_{L}^{c}}{1 / 2}\right) \\
= & \Phi_{R G S} .
\end{aligned}
$$

The proof is completed.

Thus, $\Phi_{C P S}$ is expressed as $\Phi_{C P S}=\Phi_{A P S}-\Phi_{R G S}$. All measures can exist under the conditions of $\left\{p_{i j}+p_{i^{*} j^{*}}>0\right\}, \Delta_{U}>0$ and $\Delta_{L}>0$. Then, we obtain $0 \leq \Phi_{A P S} \leq 1$ and $0 \leq \Phi_{R G S}<1$ (note that $\Phi_{R G S} \neq 1$ because of both $\Delta_{U}>0$ and $\Delta_{L}>0$ ). Since $\Phi_{C P S} \geq 0$, we obtain $0 \leq \Phi_{C P S} \leq 1$. Besides, (i) $\Phi_{C P S}=0$ if and only if there is a structure of CPS in the square table, and (ii) $\Phi_{C P S}=1$ if and only if $\Phi_{A P S}=1$ and $\Phi_{R G S}=0$; i.e., $p_{i j}=0\left(\right.$ then $\left.p_{i^{*} j^{*}}>0\right)$ or $p_{i^{*} j^{*}}=0\left(\right.$ then $\left.p_{i j}>0\right)$ for $i+j<r+1$ and $\Delta_{U}=\Delta_{L}$.

Consider the artificial probabilities in Table 1 . We see in Table 1 a that there is the structures of $p_{i^{*} j^{*}}=0\left(\right.$ then $\left.p_{i j}>0\right)$ for all $i+j<r+1$ and $\Delta_{L}=0$ (then $\Delta_{U}>0$ ). Since the degrees of departure from APS (RGS) are largest, the values of $\Phi_{A P S}$ and $\Phi_{R G S}$ are both 1 . We also see in Table la that there is not the structure of $\Delta_{L}>0$. Thus, the value of $\Phi_{C P S}$ is not definded. We see in Table $1 \mathrm{~b}$ that there is the structure of $p_{i j}=0$ or $p_{i^{*} j^{*}}=0$ for all $i+j<r+1$. Since the degrees of departure from APS are largest, the value of $\Phi_{A P S}$ is 1 . Also, we see in Table $1 \mathrm{~b}$ that there is the structure of $\Delta_{U}=\Delta_{L}(=0.3)$. Thus, the value of $\Phi_{R G S}$ is 0 . From Theorem 1 , we obtain the value of $\Phi_{C P S}$ is 1 . We see in Table 1c that there is the structure of $p_{i j}=3 p_{i^{*} j^{*}}$ for all $i+j<r+1$, thus, the CPS model hold. The value of $\Phi_{C P S}$ is 0 and the values of the $\Phi_{A P S}$ and $\Phi_{R G S}$ are both 0.189 .

\section{APPROXIMATE CONFIDENCE INTERVALS FOR MEASURES}

Let $n_{i j}$ denote the observed frequency in the $i$ th row and $j$ th column of the table $(1 \leq i, j \leq r)$. Assuming that a multinomial distribution applies to the $r \times r$ table, we shall consider approximate standard errors and large-sample confidence intervals for $\Phi_{A P S}$, $\Phi_{R G S}$ and $\Phi_{C P S}$ using the delta method of which descriptions are given by Bishop et al. (2, Sec.14.6) and Agresti (5, Sec.12.1). The sample version of $\Phi_{A P S}\left(\Phi_{R G S}, \Phi_{C P S}\right)$, i.e., $\hat{\Phi}_{A P S}\left(\hat{\Phi}_{R G S}, \hat{\Phi}_{C P S}\right)$, is given by $\Phi_{A P S}\left(\Phi_{R G S}, \Phi_{C P S}\right)$ with $\left\{p_{i j}\right\}$ replaced by $\left\{\hat{p}_{i j}\right\}$, where $\hat{p}_{i j}=n_{i j} / n$ and $n=\Sigma_{i=1}^{r} \Sigma_{j=1}^{r} n_{i j}$. Using the delta method, each of $\sqrt{n}\left(\hat{\Phi}_{A P S}-\Phi_{A P S}\right), \sqrt{n}\left(\hat{\Phi}_{R G S}-\Phi_{R G S}\right)$ and $\sqrt{n}\left(\hat{\Phi}_{C P S}-\Phi_{C P S}\right)$ has asymptotically (as $\left.n \rightarrow \infty\right)$ a normal distribution with mean zero and the corresponding variance, as

$$
\sigma^{2}\left[\Phi_{A P S}\right]=\frac{1}{\Delta^{2}}\left(\sum_{i+j \neq r+1} \sum_{i j} \Omega_{i j}^{2}-\Delta \Phi_{A P S}^{2}\right),
$$

where

$$
\begin{gathered}
\Omega_{i j}=\frac{1}{\log 2} \log \frac{2 p_{i j}}{p_{i j}+p_{i^{*} j^{*}}}, \\
\sigma^{2}\left[\Phi_{R G S}\right]=\frac{\Delta_{U} \Delta_{L}}{(\log 2)^{2} \Delta^{3}}\left(\log \frac{\Delta_{U}}{\Delta_{L}}\right)^{2},
\end{gathered}
$$

and

$$
\sigma^{2}\left[\Phi_{C P S}\right]=\frac{1}{\Delta^{2}}\left(\sum_{i+j \neq r+1} \sum_{i j} \Psi_{i j}^{2}-\Delta \Phi_{C P S}^{2}\right),
$$

where

$$
\Psi_{i j}= \begin{cases}\frac{1}{\log 2} \log \frac{\Delta p_{i j}}{\Delta_{U}\left(p_{i j}+p_{i^{*} j^{*}}\right)}(i+j<r+1), \\ \frac{1}{\log 2} \log \frac{\Delta p_{i j}}{\Delta_{L}\left(p_{i j}+p_{i^{*} j^{*}}\right)}(i+j>r+1) .\end{cases}
$$


Table 1 Artificial probabilities.

(a)

Y

\begin{tabular}{llllll}
$X$ & $\mathbf{( 1 )}$ & $\mathbf{( 2 )}$ & $\mathbf{( 3 )}$ & $\mathbf{( 4 )}$ & Total \\
\hline$(1)$ & 0.1 & 0.1 & 0.1 & 0.1 & 0.4 \\
$(2)$ & 0.1 & 0.1 & 0.1 & 0 & 0.3 \\
$(3)$ & 0.1 & 0.1 & 0 & 0 & 0.2 \\
$(4)$ & 0.1 & 0 & 0 & 0 & 0.1 \\
Total & 0.4 & 0.3 & 0.2 & 0.1 & 1 \\
\hline
\end{tabular}

(b)

\begin{tabular}{llllll}
\hline & \multicolumn{5}{c}{$Y$} \\
\cline { 2 - 5 }$X$ & $(\mathbf{1})$ & $(\mathbf{2})$ & $(3)$ & $\mathbf{( 4 )}$ & Total \\
\hline$(1)$ & 0.1 & 0 & 0.1 & 0.1 & 0.3 \\
$(2)$ & 0 & 0 & 0.1 & 0 & 0.1 \\
$(3)$ & 0.1 & 0.1 & 0.1 & 0.1 & 0.4 \\
$(4)$ & 0.1 & 0 & 0.1 & 0 & 0.2 \\
Total & 0.3 & 0.1 & 0.4 & 0.2 & 1 \\
\hline
\end{tabular}

(c)

\begin{tabular}{llllll}
\hline & \multicolumn{5}{c}{$Y$} \\
\\
\cline { 2 - 5 }$X$ & $\mathbf{( 1 )}$ & $\mathbf{( 2 )}$ & $\mathbf{( 3 )}$ & $\mathbf{( 4 )}$ & Total \\
\hline$(1)$ & 0.075 & 0.075 & 0.075 & 0.1 & 0.325 \\
$(2)$ & 0.075 & 0.075 & 0.1 & 0.025 & 0.275 \\
$(3)$ & 0.075 & 0.1 & 0.025 & 0.025 & 0.225 \\
$(4)$ & 0.1 & 0.025 & 0.025 & 0.025 & 0.175 \\
Total & 0.325 & 0.275 & 0.225 & 0.175 & 1 \\
\hline
\end{tabular}

Let $\hat{\sigma}^{2}\left[\Phi_{A P S}\right]$ denote $\sigma^{2}\left[\Phi_{A P S}\right]$ with $\left\{p_{i j}\right\}$ replaced by $\left\{\hat{p}_{i j}\right\}$. Then $\hat{\sigma}\left[\Phi_{A P S}\right] / \sqrt{n}$ is an estimated approximate standard error for $\hat{\Phi}_{A P S}$, and $\hat{\Phi}_{A P S} \pm z_{p / 2} \hat{\sigma}\left[\Phi_{A P S}\right] / \sqrt{n}$ is an approximate $100(1-p)$ percent confidence interval for $\Phi_{A P S}$, where $z_{p / 2}$ is the percentage point from the standard normal distribution corresponding to a two-tail probability equal to $p$. In a similar way, approximate confidence intervals for $\Phi_{R G S}$ and $\Phi_{C P S}$ are given.

\section{RELATIONSHIPS BETWEEN MEASURE AND LIKELIHOOD RATIO STATISTIC}

Let $G_{A P S}^{2}$ denote the likelihood ratio chi-squared statistic for testing the goodness-of-fit of the APS model, i.e.,

$$
G_{A P S}^{2}=2 n \sum_{i=1}^{r} \sum_{j=1}^{r} \hat{p}_{i j} \log \frac{\hat{p}_{i j}}{\hat{p}_{i j}^{A P S}},
$$

where

$$
\hat{p}_{i j}^{A P S}=\left\{\begin{array}{l}
\frac{1}{2}\left(\hat{p}_{i j}+\hat{p}_{i^{*} j^{*}}\right) \quad(i+j \neq r+1), \\
\hat{p}_{i j} \quad(i+j=r+1) .
\end{array}\right.
$$

Note that $\left\{\hat{p}_{i j}^{A P S}\right\}$ are the maximum likelihood estimates of $\left\{p_{i j}\right\}$ under the APS model. Then it is that the estimated measure $\hat{\Phi}_{A P S}$ is equal to $G_{A P S}^{2} / n^{\dagger}$, where $n^{\dagger}=(2 \log 2) \sum_{i+j \neq r+1} \sum_{i j}$. 
Next, let $G_{R G S}^{2}$ denote the likelihood ratio chi-squared statistic for testing the goodness-of-fit of the RGS model, i.e.,

$$
G_{R G S}^{2}=2 n \sum_{i=1}^{r} \sum_{j=1}^{r} \hat{p}_{i j} \log \frac{\hat{p}_{i j}}{\hat{p}_{i j}^{R G S}},
$$

where

$$
\hat{p}_{i j}^{R G S}= \begin{cases}\frac{\hat{\Delta}_{U}+\hat{\Delta}_{L}}{2 \hat{\Delta}_{U}} \hat{p}_{i j} & (i+j<r+1), \\ \frac{\hat{\Delta}_{U}+\hat{\Delta}_{L}}{2 \hat{\Delta}_{L}} \hat{p}_{i j} & (i+j>r+1), \\ \hat{p}_{i j} \quad(i+j=r+1) . & \end{cases}
$$

Note that $\left\{\hat{p}_{i j}^{R G S}\right\}$ are the maximum likelihood estimates of $\left\{p_{i j}\right\}$ under the RGS model. Then it is that the estimated measure $\hat{\Phi}_{R G S}$ is equal to $G_{R G S}^{2} / n^{\dagger}$.

Moreover, let $G_{C P S}^{2}$ denote the likelihood ratio chi-squared statistic for testing the goodness-of-fit of the CPS model, i.e.,

$$
G_{C P S}^{2}=2 n \sum_{i=1}^{r} \sum_{j=1}^{r} \hat{p}_{i j} \log \frac{\hat{p}_{i j}}{\hat{p}_{i j}^{C P S}},
$$

where

$$
\hat{p}_{i j}^{C P S}= \begin{cases}\frac{\hat{\Delta}_{U}}{\hat{\Delta}}\left(\hat{p}_{i j}+\hat{p}_{i^{*} j^{*}}\right) & (i+j<r+1), \\ \frac{\hat{\Delta}_{L}}{\hat{\Delta}}\left(\hat{p}_{i j}+\hat{p}_{i^{*} j^{*}}\right) & (i+j>r+1), \\ \hat{p}_{i j} \quad(i+j=r+1),\end{cases}
$$

and $\hat{\Delta}_{U}, \hat{\Delta}_{L}$ and $\hat{\Delta}$ denote $\Delta_{U}, \Delta_{L}$ and $\Delta$ with $\left\{p_{i j}\right\}$ replaced by $\left\{\hat{p}_{i j}\right\}$, respectively. Note that $\left\{\hat{p}_{i j}^{C P S}\right\}$ are the maximum likelihood estimates of $\left\{p_{i j}\right\}$ under the CPS model. Then it is that the estimated measure $\hat{\Phi}_{C P S}$ is equal to $G_{C P S}^{2} / n^{\dagger}$.

\section{EXAMPLE}

Consider the data in Tables 2 and 3, taken from Tomizawa [16]. Table 2 is constructed from the data of the unaided distance vision of 4746 students aged 18 to about 25, including about $10 \%$ of the women of the Faculty of Science and Technology, Science University of Tokyo in Japan examined in April, 1982. Table 3 is constructed from the data of the unaided distance vision of 3168 pupils aged 6-12, including about half the girls at elementary schools in Tokyo, Japan examined in June, 1984. In Tables 2 and 3 the row variable is the right eye grade and the column variable is the left eye grade with the categories ordered from the lowest grade (1) to the highest grade (4). For Tables 2 and 3 , we are interested in whether models of various PS hold. For example, when the RGS model does not hold, the probability that the sum of the right eye grade and left eye grade is 4 or less, is not equal to the probability is 6 or above. When the model does not hold, we are interested in measuring and comparing the degrees of departure from the models for Tables 2 and 3. Table 4 gives the estimates of the measures $\Phi_{A P S}$, $\Phi_{R G S}$ and $\Phi_{C P S}$, the estimated approximate standard errors for $\hat{\Phi}_{A P S}, \hat{\Phi}_{R G S}$ and $\hat{\Phi}_{C P S}$ and the approximate $95 \%$ confidence intervals for $\Phi_{A P S}$, $\Phi_{R G S}$ and $\Phi_{C P S}$.

Table 2 Unaided distance vision of 4746 students aged 18 to about 25 including about 10\% women in Faculty of Science and Technology, Science University of Tokyo in Japan examined in April 1982; from Tomizawa [16].

\begin{tabular}{lccccc}
\hline \multicolumn{7}{c}{ Left eye grade } \\
\hline Right eye grade & Lowest (1) & Second (2) & Third (3) & Highest (4) & Total \\
\hline Lowest (1) & 1429 & 249 & 25 & 20 & 1723 \\
Second (2) & 185 & 660 & 124 & 64 & 1033 \\
Third (3) & 23 & 114 & 221 & 149 & 507 \\
Highest (4) & 22 & 40 & 130 & 1291 & 1483 \\
Total & 1659 & 1063 & 500 & 1524 & 4746 \\
\hline
\end{tabular}


Table 3 Unaided distance vision of 3168 pupils aged 6-12 including about half girls at elementary schools in Tokyo examined in June 1984; from Tomizawa [16].

\begin{tabular}{lccccc}
\hline \multicolumn{7}{c}{ Left eye grade } \\
\hline Right eye grade & Lowest (1) & Second (2) & Third (3) & Highest (4) & Total \\
\hline Lowest (1) & 92 & 16 & 7 & 12 & 127 \\
Second (2) & 15 & 75 & 42 & 10 & 142 \\
Third (3) & 5 & 33 & 138 & 96 & 272 \\
Highest (4) & 10 & 21 & 126 & 2470 & 2627 \\
Total & 122 & 145 & 313 & 2588 & 3168 \\
\hline
\end{tabular}

Table 4 Estimate of $\Phi_{A P S}, \Phi_{R G S}$ and $\Phi_{C P S}$, estimated approximate standard error for $\hat{\Phi}_{A P S}, \hat{\Phi}_{R G S}$ and $\hat{\Phi}_{C P S}$, and approximate $95 \%$ confidence interval for $\Phi_{A P S}, \Phi_{R G S}$ and $\Phi_{C P S}$, applied to Tables 2 and 3 .

\begin{tabular}{lcccc}
\hline Applied data & $\hat{\boldsymbol{\Phi}}$ & Estimated measure & Standard error & Confidence interval \\
\hline Table 2 & $\hat{\Phi}_{A P S}$ & 0.049 & 0.005 & $(0.038,0.059)$ \\
& $\hat{\Phi}_{R G S}$ & 0.017 & 0.003 & $(0.010,0.023)$ \\
& $\hat{\Phi}_{C P S}$ & 0.032 & 0.004 & $(0.023,0.041)$ \\
Table 3 & $\hat{\Phi}_{A P S}$ & 0.693 & 0.016 & $(0.662,0.724)$ \\
& $\hat{\Phi}_{R G S}$ & 0.640 & 0.017 & $(0.607,0.674)$ \\
& $\hat{\Phi}_{C P S}$ & 0.053 & 0.008 & $(0.037,0.069)$ \\
\hline
\end{tabular}

From Table 4, when the degrees of departure from APS for Tables 2 and 3 are compared using the confidence interval for $\Phi_{A P S}$, it would be greater in Table 3 than in Table 2. The same can be said about the degrees of departure from RGS. However, the comparison between degrees of departure from CPS in Tables 2 and 3 may be impossible. Because the values in the confidence interval for Table 3 are not always greater than the values in the confidence interval for Table 2.

\section{CONCLUDING REMARKS}

The measures $\Phi_{A P S}, \Phi_{R G S}$ and $\Phi_{C P S}$ always range between 0 and 1 independent of the dimension $r$ and sample size $n$. So, these measures may be useful for comparing the degrees of departure from APS, RGS and CPS in several tables, respectively.

As is well known, in general, the absolute value of the correlation coefficient between two variables is theoretically 0 or more and 1 or less. However in many actual data, the estimated absolute value of the correlation coefficient is 0 or more and less than 1 . Similarly, each of the proposed measures theoretically ranges between 0 and 1 . However, when the value of the proposed measure is 1 , it has some structures of probability zero. We note that in many actual data, the estimated value of the measures is 0 or more and less than 1 .

The measure $\Phi_{A P S}$ is used to measure what degree the departure from the APS model is toward the maximum departure of APS defined in Section 2. Similarly, the measure $\Phi_{R G S}\left(\Phi_{C P S}\right)$ is used to measure what degree the departure from the RGS model (the CPS model) is toward the maximum departure of RGS (CPS) defined in Section 2. We note that the definitions of the three models and the corresponding maximum departures are different. That is, we point out that the purpose of using each measure is different. Also, from Theorem 1 , note that the values of the three measures are related to each other.

The CPS model imposes no restriction on the reverse diagonal cell probabilities $\left\{p_{i i^{*}}\right\}$. Thus, the structure of CPS based on the probabilities $\left\{p_{i j}\right\}$, i.e., $p_{i j} / p_{i^{*} j^{*}}=\tau(i+j<r+1)$, is also expressed as $p_{i j}^{c} / p_{i^{*} j^{*}}^{c}=\tau$, using the conditional cell probabilities $\left\{p_{i j}^{c}\right\}, i+j \neq r+1$. So, it seems natural that the measure of the degree of departure from CPS and their ranges do not depend on the reverse diagonal cell probabilities. In the sample versions, it may seem to many readers that both measures $G_{C P S}^{2} / n$ and $\hat{\Phi}_{C P S}$ are reasonable measures for representing the degree of departure from CPS. However, $\hat{\Phi}_{C P S}$ rather than $G_{C P S}^{2} / n$ would be useful for comparing the degree of departure from CPS in several tables. Because the range of $G_{C P S}^{2} / n$ depends on the reverse diagonal proportions, i.e., $0 \leq\left(G_{C P S}^{2} / n\right) \leq\left(n^{\dagger} / n\right)\left[=(2 \log 2)\left(1-\Sigma_{i=1}^{r} n_{i i^{*}} / n\right)\right]$, but $\hat{\Phi}_{C P S}$ always ranges between 0 and 1 without depending on the reverse diagonal proportions. By a similar reason, $\hat{\Phi}_{C P S}$ may also be preferable to $G_{C P S}^{2}$ for comparing them. The same can be said about $\hat{\Phi}_{A P S}$ and $\hat{\Phi}_{R G S}$.

Note that the proposed three measures cannot be used to test the goodness-of-fit of each model. Also note that the three measures have different purposes and it is meaningless to compare the values of the three measures. Kurakami et al. [8] gave the orthogonality of likelihood ratio chi-square statistics for testing the goodness-of-fit, i.e., $G_{A P S}^{2}=G_{C P S}^{2}+G_{R G S}^{2}$. We note that Theorem 1 is corresponding to the population version of this orthogonality. 
We could extend the proposed measure $\Phi_{A P S}$ to the power-divergence type measure, as

$$
\Phi_{A P S}^{(\lambda)}=\frac{\lambda(\lambda+1)}{2\left(2^{\lambda}-1\right)} I_{A P S}^{(\lambda)} \quad \text { for } \lambda>-1,
$$

where

$$
\left.I_{A P S}^{(\lambda)}=\sum_{i+j \neq r+1} \sum_{p_{i j}^{c}}\left\{\left(\frac{p_{i j}^{c}}{p_{i j}^{A P S}}\right)^{\lambda}-1\right\}\right],
$$

and the value at $\lambda=0$ is taken to be the limit as $\lambda \rightarrow 0$. Thus, $\Phi_{A P S}^{(0)}$ is identical to the measure $\Phi_{A P S}$. Similarly, we could extend the proposed measures $\Phi_{R G S}\left(\Phi_{C P S}\right)$ to the power-divergence type measures. For the detail of the power-deivergence, see Read and Cressie ([17], p.15). However, for any $\lambda(\lambda \neq 0)$, we note that the value of the power-divergence type measure $\Phi_{A P S}^{(\lambda)}$ is not equal to the sum of the value of the measure $\Phi_{R G S}^{(\lambda)}$ and the value of the measure $\Phi_{C P S}^{(\lambda)}$.

\section{CONFLICTS OF INTEREST}

The authors declare that there are no conflicts of interest regarding the publication of this paper.

\section{AUTHORS' CONTRIBUTIONS}

All authors contributed equally to the writing of this paper. All authors have read and agreed to the published version of the manuscript.

\section{Funding Statement}

There is no funding of this paper.

\section{ACKNOWLEDGMENTS}

The authors would like to thank the editor and the three referees for their helpful comments.

\section{REFERENCES}

1. A.H. Bowker, J. Am. Stat. Assos. 43 (1948), 572-574.

2. Y.M.M. Bishop, S.E. Fienberg, P.W. Holland, Discrete Multivariate Analysis: Theory and Practice, The MIT Press, Cambridge, 1975.

3. C.B. Read, Commun. Stat. - Theory Methods. 6 (1977), 553-562.

4. P. McCullagh, Biom. 65 (1978), 413-418.

5. A.Agresti, Categorical Data Analysis, Wiley, New York, 1990.

6. S. Tomizawa, Biom. 49 (1993), 993-887.

7. K. D. Wall, G. A. Lienert, Biom. J. 18 (1976), 259-264.

8. H. Kurakami, K. Negishi, S. Tomizawa, J. Stat. Adv. Theory Appl. 17 (2017), 33-42.

9. S. Tomizawa, Biom. J. 28 (1986), 387-393.

10. C.R. Rao, Bull. Int. Stat. Instit. 34 (1954), 90-97.

11. F. Mosteller, J. Am. Stat. Assoc. 63 (1968), 1-28.

12. Y. J. Wang, Commun. Stat. Simul. C. 41 (2012), 32-43.

13. S. Tomizawa, Stat. Sin. 4 (1994), 325-334.

14. S. Tomizawa, Behaviormetrika. 22 (1995), 91-98.

15. S. Tomizawa, K. Saitoh, Calcutta Stat. Assoc. Bull. 49 (1999), 32-39.

16. S. Tomizawa, Environ. Health Perspect. 63 (1985), 235-239.

17. T.R.C. Read, N.A.C. Cressie, Goodness-of-Fit Statistics for Discrete Multivariate Data, Springer-Verlag, New York, 1988. 